\section{Extrinsische Schlafstörungen}

Helga Peter ${ }^{1}$ und Thomas Penzel ${ }^{2}$

${ }^{1}$ Marburg, Deutschland

${ }^{2}$ Interdisziplinäres Schlafmedizinisches Zentrum, Charité Universitätsmedizin Berlin, Berlin, Deutschland

\section{Synonyme}

Extrinsische Dyssomnien

\section{Englischer Begriff}

extrinsic sleep disorders

\section{Definition}

Der Begriff diente in der ersten Version der ICSD von 1990 als Bezeichnung für Schlafstörungen, die auf von außen einwirkenden Ursachen beruhen, wozu auch das Verhalten gezählt wird. Die Hauptbeschwerden sind aber keineswegs ausschließlich insomnischer Natur. Häufig steht analog zu den Verhältnissen bei den Zirkadianen Schlaf-Wach-Rhythmusstörungen die hypersomnische Symptomatik mit der Hauptbeschwerde Tagesschläfrigkeit im Vordergrund.

Siehe auch

- „Diagnostische Klassifikationssysteme“

- „Extrinsische Insomnien“

- „Umgebungsbedingte Schlafstörung“ 